\title{
REMARKS ON THE PRANDTL EQUATION FOR A PERMEABLE WALL
}

\author{
R. TEMAM ${ }^{1,2}$ AND X. WANG ${ }^{3}$
}

\begin{abstract}
The goal of this article is to study the boundary layer for a flow in a channel with permeable walls. Observing that the Prandtl equation can be solved almost exactly in this case, we are able to derive rigorously a number of results concerning the boundary layer and the convergence of the Navier-Stokes equations to the Euler equations. We indicate also how to derive higher order terms in the inner and outer expansions with respect to the kinematic viscosity $\nu$.
\end{abstract}

\section{Contents}

1. Introduction

2. The Prandtl type equations 3

3. Validity of the asymptotic expansion 9

4. Discussion on stability 12

Appendix $\quad 15$

$\begin{array}{ll}\text { Acknowledgments } & 17\end{array}$

References 17

\section{INTRODUCTION}

The behavior of the solutions to the Navier-Stokes equations at vanishing viscosity (large Reynolds numbers) is an outstanding open problem both in fluid mechanics and in mathematical analysis. In the physically realistic case of wall bounded flows, two phenomena occur: on the one hand, inside the flow, the cascading of energy between large eddies and

Date: May 10, 2000.

Key words and phrases. Boundary layers, Fluid Mechanics, Vanishing viscosity, Navier-Stokes equations, Euler equations, Prandtl equations.

${ }^{1}$ Laboratoire d'Analyse Numérique, Université Paris Sud, Batiment 425, 91405 Orsay, France.

${ }^{2}$ Institute for Scientific Computing \& Applied Mathematics, Indiana University, Rawles Hall, Bloomington, IN 47405-5701, USA, temam@indiana.edu.

${ }^{3}$ Department of Mathematics, Iowa State University, Ames, IA 50011, USA, wang@math.iastate.edu. 
small eddies and its dissipation by viscosity; on the other hand, at the boundary and in the boundary layer, which may be turbulent vortices are generated which drive the flow. In this article, we are interested, in the incompressible case, in the question of how well the Navier-Stokes equations are approximated by the corresponding Euler equations.

In mechanical engineering an abundant literature is devoted to the study of boundary layers following the pioneering work of L. Prandtl (1905); see e.g. R. Balian and J. L. Peube (1977), and other articles in this volume. On the mathematical side in the context of modern functional analysis, and for incompressible fluids, there is a more limited literature. O. Oleinik (1963) addresses the mathematical theory of the Prandtl equations themselves (see also the more recent book O. Oleinik and V. N. Samokhin (1999)); M. I. Vishik and L. A. Lyusternik (1957), J. L. Lions (1973) address many boundary layer issues in the context of other areas of sciences and engineering. In his article J. L. Lions introduces the concept of correctors, which we use; the point of view is then slightly different from the traditional point of view in boundary layer theory.

In this article we want to report on a work in progress investigating a number of issues in that direction (see R. Temam and X. Wang (2000), hereafter referred to as [TW] and other articles in preparation). In [TW] we consider the case where the boundary is noncharacteristic; more precisely we consider the flow in a channel with permeable boundary. In this case the Prandtl equation can be solved as we recall below, and we are able to establish a number of convergence results of the viscous solutions to the inviscid ones. This is the object of Section 2. In Section 3, continuing this line of thought, we study higher order approximations, and speculate on the form of the higher order terms in an inner-outer expansion with respect to the kinematic viscosity $\nu$; the validity of such expansions will be addressed elsewhere. Finally, in Section 4, we briefly discuss another important issue, namely the stability of the boundary layer, a problem which corresponds to its actual occurrence in the physical reality. We emphasize the fact that with our presentation based on the use of correctors, we do not need the technic of matched asymptotic expansions, although we do use stretched coordinates in the boundary layer. 


\section{The Prandtl type equations}

We consider the Navier-Stokes equations for incompressible Newtonian fluids in a channel with injection and suction:

$$
\begin{aligned}
\frac{\partial u^{\nu}}{\partial t}+\left(u^{\nu} \nabla\right) u^{\nu}-\nu \Delta u^{\nu}+\nabla p^{\nu} & =f \quad \text { in } \Omega \times(0, T), \\
\operatorname{div} u^{\nu} & =0 \quad \text { in } \Omega \times(0, T), \\
u^{\nu} & =u_{0} \quad \text { at } t=0, \\
u^{\nu} & =v_{\text {top }} \quad \text { at } z=0, \\
u^{\nu} & =v_{\text {bot }} \quad \text { at } z=h .
\end{aligned}
$$

Here $\Omega$ is the channel, $\Omega=\left(0, L_{1}\right) \times\left(0, L_{2}\right) \times(0, h)$, with periodicity assumed in $x$ (period $\left.L_{1}\right)$ and $y\left(\right.$ period $\left.L_{2}\right)$ for all involved functions.

We would assume a porous boundary, with injection at the top of the channel

$$
v_{\text {top }} \cdot \vec{n} \leq-\beta<0 \quad \text { at } \quad z=h,
$$

and suction at the bottom of the channel,

$$
v_{\text {bot }} \cdot \vec{n} \geq \beta>0 \quad \text { at } z=0,
$$

where $\vec{n}$ is the unit outward normal to $\partial \Omega$. The usual compatibility condition

$$
\int_{z=h} v_{\mathrm{top}} \cdot \vec{n}+\int_{z=0} v_{\mathrm{bot}} \cdot \vec{n}=0
$$

will be understood.

These boundary conditions are sometimes referred to as non-characteristic boundary conditions since the streamlines are transversal to the boundary.

The global existence of Leray-Hopf weak solution to the Navier-Stokes system is wellknown provided the boundary, initial and external forcing data are smooth enough. (The existence of steady state solution is largely open though) In the two dimensional case (with the dependence on $y$ suppressed) the existence and uniqueness of smooth solution is easy to derive by mimicking classical approaches, provided the data are smooth and certain compatibility conditions are satisfied.

Our interest here is in the asymptotic behavior of the solutions of the Navier-Stokes equations at small kinematic viscosity $\nu$. 
A naive approach is to treat this as a regular perturbation problem and to seek for a sequence of approximations of the form

$$
u^{\nu} \sim \sum_{j=0}^{\infty} \nu^{j} u^{j}
$$

Inserting this into the Navier-Stokes equations, collecting terms of the same order in $\nu$ we obtain,

$$
\begin{aligned}
\frac{\partial u^{0}}{\partial t}+\left(u^{0} \cdot \nabla\right) u^{0}+\nabla p^{0} & =f \text { in } \Omega \times(0, T), \\
\operatorname{div} u^{0} & =0 \text { in } \Omega \times(0, T), \\
u^{0} & =u_{0} \text { at } t=0, \\
\frac{\partial u^{1}}{\partial t}+\left(u^{0} \cdot \nabla\right) u^{1}+\left(u^{1} \cdot \nabla\right) u^{0}+\nabla p^{1} & =\Delta u^{0} \text { in } \Omega \times(0, T), \\
\operatorname{div} u^{1} & =0 \text { in } \Omega \times(0, T), \\
u^{1} & =0 \text { at } t=0, \\
\frac{\partial u^{j}}{\partial t}+\left(u^{0} \cdot \nabla\right) u^{j}+\left(u^{j} \cdot \nabla\right) u^{0}+\nabla p^{j} & =\Delta u^{j-1}-\sum_{l=1}^{j-1}\left(u^{l} \cdot \nabla\right) u^{j-l} \text { in } \Omega \times(0, T), \\
\operatorname{div} u^{j} & =0 \text { in } \Omega \times(0, T), \\
u^{j} & =0 \text { at } t=0 .
\end{aligned}
$$

In the case without physical boundary, i.e., $\Omega=\mathbb{R}^{n}$ with decay at infinity or periodic boundary condition in all directions, the validity of the asymptotic expansion can be easily verified, at least for smooth data.

The zeroth order is exactly the Euler equations for incompressible inviscid fluids.

In the case of flow in a limited region we need to specify boundary conditions for the $u^{j}$ at all orders.

If the boundary is impermeable, the boundary conditions for the $u^{j}$ 's are

$$
u^{j} \cdot \vec{n}=0 \text { on } \partial \Omega
$$


If the boundary is porous, we have to specify the whole velocity where the flow is entering $(z=h)$, and the normal velocity where the flow is going out $(z=0)$ :

$$
\begin{aligned}
u^{0} & =v_{\text {top }} \text { at } z=h, \\
u^{0} \cdot \vec{n} & =v_{\text {bot }} \cdot \vec{n} \text { at } z=0, \\
u^{j} & =0 \text { at } z=h, j \geq 1, \\
u^{j} \cdot \vec{n} & =0 \text { at } z=0, j \geq 1 .
\end{aligned}
$$

This expansion is sometimes referred to as the outer (outside of the boundary layer) expansion for $u^{\nu}$.

Notice that, in general, $\sum_{j=0}^{n} \nu^{j} u^{j}$ cannot match $u^{\nu}$ at the outlet boundary $z=0$. Thus such asymptotic expansions cannot be valid uniformly, in the whole domain (i.e. not up to the boundary). Thus various boundary layer correction terms are needed (which we call correctors) in order to obtain convergence up to the boundary. Hence we propose a new sequence of approximations with correctors:

$$
u^{\nu} \sim \sum_{j=0}^{\infty} \nu^{j}\left(u^{j}+\theta^{j}\right)
$$

Here we deviated from Prandtl's classical approach or more generally from the matched asymptotic approach where one does inner expansions (within the boundary layer) using stretched coordinates on the viscous solution $u^{\nu}$ directly and do later the matching with the outer solution. The alternative treatment we present here, the so-called corrector approach (see Vishik and Lyusternik (1957), Lions (1973)), uses the stretched coordinate argument later on, to compute or approximate the correctors $\theta^{j}$.

Utilizing the stretched coordinate

$$
Z=\frac{z}{\nu}
$$


and the ansatz of new sequence of approximation with corrector, we have formally

$$
\begin{aligned}
\sum_{j=0}^{\infty} \nu^{j} \frac{\partial u^{j}}{\partial t} & +\sum_{j=0}^{\infty} \nu^{j} \frac{\partial \theta^{j}}{\partial t}+\sum_{j=0}^{\infty} \nu^{j} \sum_{l=0}^{j}\left(\left(u^{\ell} \cdot \nabla\right) u^{j-\ell}+\left(\theta^{\ell} \cdot \nabla\right) u^{j-\ell}\right) \\
& +\sum_{j=0}^{\infty} \nu^{j} \sum_{\ell=0}^{j}\left(\left(u_{\tau}^{\ell}+\theta_{\tau}^{\ell}\right) \cdot \nabla_{\tau}\right) \theta^{j-\ell} \\
& +\sum_{j=-1}^{\infty} \nu^{j} \sum_{\ell=0}^{j+1} u_{3}^{\ell} \frac{\partial \theta^{j+1-\ell}}{\partial Z}+\sum_{j=0}^{\infty} \nu^{j-1} \sum_{\ell=0}^{j} \theta_{3}^{\ell} \frac{\partial \theta^{j-\ell}}{\partial Z} \\
& -\sum_{j=1}^{\infty} \nu^{j} \Delta u^{j-1}-\sum_{j=1}^{\infty} \nu^{j} \Delta_{\tau} \theta^{j-1} \\
& -\sum_{j=-1}^{\infty} \nu^{j} \frac{\partial^{2} \theta^{j+1}}{\partial Z^{2}}+\nabla p=f, \\
& \operatorname{div} \theta^{j}=0
\end{aligned}
$$

where $\Delta_{\tau}, \nabla_{\tau}, \theta_{\tau}, u_{\tau}$ represent the tangential Laplacian, tangential gradient, tangential component(s) of $\theta$ and $u$ respectively.

Utilizing the stretched coordinates, the incompressibility condition can be written as:

$$
\frac{\partial \theta_{1}^{j}}{\partial x}+\frac{\partial \theta_{2}^{j}}{\partial y}+\frac{1}{\nu} \frac{\partial \theta_{3}^{j}}{\partial Z}=0
$$

Hence $\frac{1}{\nu} \theta_{3}^{j}$ is expected to be bounded.

Thus, if we group terms with the same order we obtain:

\section{At order -1:}

$$
-\frac{1}{\nu}\left(\frac{\partial^{2} \theta^{0}}{\partial Z^{2}}-u_{3}^{0} \frac{\partial \theta^{0}}{\partial Z}\right)+\nabla q^{0}=0,
$$

which can be further approximated by

$$
-\frac{1}{\nu}\left(\frac{\partial^{2} \theta^{0}}{\partial Z^{2}}-\left.u_{3}^{0}\right|_{z=0} \frac{\partial \theta^{0}}{\partial Z}\right)+\nabla q^{0}=0
$$

The boundary condition is given by

$$
\begin{aligned}
& \theta^{0}=0 \text { at } z=h, \\
& \theta_{3}^{0}=0 \text { at } z=0, \\
& \theta_{k}^{0}=-u_{k}^{0} \text { at } z=0, \quad k=1,2 .
\end{aligned}
$$




\section{At order 0:}

$$
\begin{aligned}
\frac{\partial u^{0}}{\partial t} & +\frac{\partial \theta^{0}}{\partial t}+\left(u^{0} \cdot \nabla\right) u^{0}+\left(\theta^{0} \cdot \nabla\right) u^{0}+\left(\left(u_{\tau}^{0}+\theta_{\tau}^{0}\right) \cdot \nabla_{\tau}\right) \theta^{0} \\
& +\frac{1}{\nu} \theta_{3}^{0} \frac{\partial \theta_{3}^{0}}{\partial Z}+u_{3}^{0} \frac{\partial \theta^{1}}{\partial Z}+u_{3}^{1} \frac{\partial \theta^{0}}{\partial Z}-\frac{\partial^{2} \theta^{1}}{\partial Z^{2}}+\nabla q^{1}=f
\end{aligned}
$$

Utilizing the equations for $u^{0}$ we have (with the pressure term replaced by a new one if necessary)

$$
\begin{aligned}
& -\frac{\partial^{2} \theta^{1}}{\partial Z^{2}}+u_{3}^{0} \frac{\partial \theta^{1}}{\partial Z}+\nabla q^{1}=-\frac{\partial \theta^{0}}{\partial t}-\left(\theta^{0} \cdot \nabla\right) u^{0}-\left(\left(u_{\tau}^{0}+\theta_{\tau}^{0}\right) \cdot \nabla_{\tau}\right) \theta^{0}-u_{3}^{1} \frac{\partial \theta^{0}}{\partial Z}-\frac{1}{\nu} \theta_{3}^{0} \frac{\partial \theta_{3}^{0}}{\partial Z}, \\
& \operatorname{div} \theta^{1}=0 \\
& \theta^{1}=0 \text { at } z=h, \theta_{3}^{1}=0 \text { at } z=0, \theta_{k}^{1}=-u_{k}^{1} \text { at } z=0, k=1,2 .
\end{aligned}
$$

At order $j(j \geq 1)$ :

$$
\begin{aligned}
\frac{\partial u^{j}}{\partial t} & +\frac{\partial \theta^{j}}{\partial t}+\sum_{\ell=0}^{j}\left(\left(u^{\ell} \cdot \nabla\right) u^{j-\ell}+\left(\theta^{\ell} \cdot \nabla\right) u^{j-\ell}\right) \\
& +\sum_{\ell=0}^{j}\left(\left(u_{\tau}^{\ell}+\theta_{\tau}^{\ell}\right) \cdot \nabla_{\tau}\right) \theta^{j-\ell} \\
& +\sum_{\ell=0}^{j+1} u_{3}^{\ell} \frac{\partial \theta^{j+1-\ell}}{\partial Z}+\sum_{\ell=0}^{j} \frac{\theta_{3}^{\ell}}{\nu} \frac{\partial \theta^{j-\ell}}{\partial Z} \\
& -\Delta u^{j-1}-\Delta_{\tau} \theta^{j-1}-\frac{\partial^{2} \theta^{j+1}}{\partial Z^{2}}+\nabla q^{j+1}=0 .
\end{aligned}
$$


Utilizing the equation for $u^{j}$ we deduce

$$
\begin{aligned}
-\frac{\partial^{2} \theta^{j+1}}{\partial Z^{2}}+u_{3}^{0} \frac{\partial \theta^{j+1}}{\partial Z}+\nabla q^{j+1}= & -\frac{\partial \theta^{j}}{\partial t}-\sum_{\ell=0}^{j}\left(\theta^{\ell} \cdot \nabla\right) u^{j-\ell} \\
& -\sum_{\ell=0}^{j}\left(\left(u_{\tau}^{\ell}+\theta_{\tau}^{\ell}\right) \cdot \nabla_{\tau}\right) \theta^{j-\ell}-\sum_{\ell=1}^{j+1} u_{3}^{\ell} \frac{\partial \theta^{j+1-\ell}}{\partial Z} \\
& -\sum_{\ell=0}^{j} \frac{\theta_{3}^{\ell}}{\nu} \frac{\partial \theta^{j-\ell}}{\partial Z}+\Delta_{\tau} \theta^{j-1}, \\
\operatorname{div} \theta^{j+1}= & 0 \quad 0 \text { at } z=h \\
\theta^{j+1}= & \text { at } z=0 \\
\theta_{3}^{j+1}= & -u_{k}^{j+1} \text { at } z=0, \quad k=1,2 . \\
\theta_{k}^{j+1}= &
\end{aligned}
$$

These equations for correctors can be further approximated by

$$
\begin{aligned}
&-\frac{\partial^{2} \theta^{j+1}}{\partial Z^{2}}+\left.u_{3}^{0}\right|_{z=0} \frac{\partial \theta^{j+1}}{\partial Z}+\nabla q^{j+1}= \\
&-\frac{\partial \theta^{j}}{\partial t}-\sum_{\ell=0}^{j}\left(\theta^{\ell} \cdot \nabla\right) u^{j-\ell}-\sum_{\ell=0}^{j}\left(\left(u_{\tau}^{\ell}+\theta_{\tau}^{\ell}\right) \cdot \nabla_{\tau}\right) \theta^{j-\ell}-\sum_{\ell=1}^{j+1} u_{3}^{\ell} \frac{\partial \theta^{j+1-\ell}}{\partial Z} \\
&-\sum_{\ell=0}^{j} \frac{\theta_{3}^{\ell}}{\nu} \frac{\partial \theta^{j-\ell}}{\partial Z}+\Delta_{\tau} \theta^{j-1}+\frac{\left(\left.u_{3}^{0}\right|_{z=0}-u_{3}^{0}\right)}{\nu} \frac{\partial \theta^{j}}{\partial Z} \text { for } j \geq 1, \\
& \operatorname{div} \theta^{j+1}=0, \\
& \theta^{j+1}=0 \text { at } z=h, \\
& \theta_{3}^{j+1}=0 \text { at } z=0, \\
& \theta_{k}^{j+1}=-u_{k}^{j+1} \text { at } j=0, k=1,2 .
\end{aligned}
$$

These equations for $\theta^{j}$ can be viewed as Prandtl's type equations since they are derived in exactly the same spirit as in Prandtl's original work of 1905.

The well-posedness of these Prandtl type equations is straightforward. The asymptotic behavior of $\theta^{j}$ is less obvious. We intend to prove elsewhere that, roughly speaking:

$$
\theta^{j}=\sum_{k=1, l=0}^{3 j+1} C_{j k l}(t, x, y, z)\left(\frac{z}{\nu}\right)^{l} \Phi(k z)+\nu C_{j}(t, x, y, z) .
$$

Here

$$
\Phi(z)=\Phi(t, x, y, z)=e^{-\left.\frac{1}{\nu} z u_{3}^{0}\right|_{z=0}}=e^{-\frac{1}{\nu} z u_{3}^{0}(t, x, y, 0)}
$$


and the $C_{j k l}$ and $C_{j}$ are smooth and bounded (in various function spaces) independently of $\nu$ provided that the data are smooth, that certain compatibility conditions are satisfied and the inviscid problems produce unique smooth solution.

The boundary conditions for the inviscid equations (at the various orders of outer expansions) are somewhat non-conventional. The usual boundary condition associated with inviscid flows is the so-called impermeable boundary condition $u^{j} \cdot \vec{n}=0$ at the boundary. However in our case of porous boundary, we need to specify the total velocity at the upwind direction; otherwise the system will be under-determined. For the local in time well-posedness of these problems one may consult, among others, the work of Antontsev, Kazhikhov and Monakhov (1990).

\section{VALIDITY OF THE ASYMPTOTIC EXPANSION}

In this section we give a sketch of the validity of the asymptotic expansion, or the Prandtl type equations that we proposed in the previous section. We give the sketch for the first term, namely $\theta^{0}$ only. For the 0 order case we follow in part R. Temam and X. Wang (2000); for the 1- order case the details will be given elsewhere.

For the 0 order Prandtl type equation, we consider the adjusted difference

$$
w^{0}=u^{\nu}-u^{0}-\theta^{0}
$$

Notice that $w^{0}$ satisfies the equation

$$
\begin{aligned}
\frac{\partial w^{0}}{\partial t}-\nu \Delta w^{0}+\left(u^{\nu} \cdot \nabla\right) w^{0}+\left(w^{0} \cdot \nabla\right) u^{0}+\left(w^{0} \cdot \nabla\right) \theta^{0}+\nabla\left(p^{\nu}-p^{0}-q^{0}\right) \\
=\frac{\partial \theta^{0}}{\partial t}+\nu \Delta u^{0}+\nu \Delta \theta^{0}-\left(\theta^{0} \cdot \nabla\right) \theta^{0}-\left(\theta^{0} \cdot \nabla\right) u^{0}-\left(u^{0} \cdot \nabla\right) \theta^{0}, \\
\operatorname{div} w^{0}=0, \\
w^{0}=0 \text { at } z=0, h .
\end{aligned}
$$

Our goal is to prove that $w^{0}$ is small in some sense. We employ the classical energy method since no easy uniform estimates on the pressure (in terms of viscosity) can be derived. 
We focus on the right-hand-side first. It can be rewritten as

$$
\begin{aligned}
\frac{\partial \theta^{0}}{\partial t} & +\nu \Delta u^{0}+\nu \Delta \theta^{0}-\left.u_{3}^{0}\right|_{z=0} \frac{\partial \theta^{0}}{\partial z}-\left(u_{\tau}^{0} \cdot \nabla_{\tau}\right) \theta^{0} \\
& -\left(\theta^{0} \cdot \nabla\right) \theta^{0}-\left(\theta^{0} \cdot \nabla\right) u^{0}-\left(u_{3}^{0}-\left.u_{3}^{0}\right|_{z=0}\right) \frac{\partial \theta^{0}}{\partial z} \\
= & \frac{\partial \theta^{0}}{\partial t}+\nu \Delta u^{0} \nu \Delta_{\tau} \theta^{0}-\left(u_{\tau}^{0} \cdot \nabla_{\tau}\right) \theta^{0}-\left(\theta^{0} \cdot \nabla\right) \theta^{0}-\left(\theta^{0} \cdot \nabla\right) u^{0}-\nabla q^{0} \\
& -\left(u_{3}^{0}-\left.u_{3}^{0}\right|_{z=0}\right) \frac{\partial \theta^{0}}{\partial z} .
\end{aligned}
$$

According to the decomposition of $\theta^{0}$ into a boundary layer part and a regular part, we have

$$
\theta^{0}=C_{010}(t, x, y) \Phi(z)+\nu C_{0}(t, x, y, z)
$$

Thus, it is easy to see that

$$
\frac{\partial \theta^{0}}{\partial t}-\left(u_{\tau}^{0} \cdot \nabla_{\tau}\right) \theta^{0}-\left(\theta_{\tau}^{0} \cdot \nabla_{\tau}\right) \theta^{0}-\left(\theta^{0} \cdot \nabla\right) u^{0}+\nu \Delta_{\tau} \theta^{0}+\nu \Delta u^{0}
$$

can be written in the same form, i.e., a boundary layer part of the form bounded function times $\Phi$ and a regular part of the form $\nu$ times a bounded function.

Notice that $u_{3}^{0}-\left.u_{3}^{0}\right|_{z=0}=0$ at $z=0$; hence

$$
\left(u_{3}^{0}-\left.u_{3}^{0}\right|_{z=0}\right) \frac{\partial \theta^{0}}{\partial z}=\frac{u_{3}^{0}-\left.u_{3}^{0}\right|_{z=0}}{z} z \frac{\partial \theta^{0}}{\partial z}
$$

could also be decomposed as a boundary layer part of the form bounded function times, $(z / \nu) \Phi$ and a regular part of the form $\nu$ times a bounded function.

For the term $\theta_{3}^{0} \frac{\partial \theta^{0}}{\partial z}$, notice that by the incompressibility the third component of the boundary layer part of the 0 order corrector $\theta^{0}$ must be such that $\frac{C_{00}}{\nu}$ is bounded. Thus $\frac{\theta_{3}^{0}}{\nu}$ is bounded. Thus $\theta_{3}^{0} \frac{\partial \theta^{0}}{\partial z}$ could also be decomposed into a boundary layer part of the form bounded function times $\Phi$ and a regular part of the form $\nu$ times a bounded function.

To summarize we write the right-hand-side (RHS) of the equation for the adjusted difference $w^{0}$ in the form

$$
\mathrm{RHS}_{1}+\mathrm{RHS}_{2}
$$

where

$$
\operatorname{RHS}_{1}=g_{1}(t, x, y, z) \Phi(t, x, y, z),
$$

with $g_{1}$ a uniformly bounded function and

$$
\mathrm{RHS}_{2}=\nu g_{2}(t, x, y, z),
$$

with $g_{2}$ a function bounded in $L^{2}$ (and $L^{\infty}$ ). 
Hence the energy estimates yields, for the right-hand-side (RHS):

$$
\begin{aligned}
\left|\int_{\Omega} \mathrm{RHS} \cdot w^{0}\right| & \leq\left|\int_{\Omega} g_{1} \Phi w^{0}\right|+\left|\int_{\Omega} \nu g_{2} w^{0}\right| \\
& \leq \nu\left|g_{2}\right|_{L^{2}}\left|w^{0}\right|_{L^{2}}+\left|g_{1}\right|_{L^{\infty}}|z \Phi|_{L^{2}}\left|\frac{w^{0}}{z}\right|_{L^{2}} \\
& \leq \kappa \nu\left|w^{0}\right|_{L^{2}}+\kappa \nu^{3 / 2}\left|\frac{\partial w^{0}}{\partial z}\right|_{L^{2}}
\end{aligned}
$$

(Thanks to Hardy's inequality)

$$
\leq \frac{\nu}{8}\left|\nabla w^{0}\right|_{L^{2}}^{2}+\frac{1}{2}\left|w^{0}\right|_{L^{2}}^{2}+\kappa \nu^{2}
$$

where $\kappa$ is a generic constant depending on the data but independent of $\nu$.

Now we go back to the left-hand-side. It is easy to see that

$$
\begin{aligned}
\int_{\Omega} \frac{\partial w^{0}}{\partial t} w^{0} & =\frac{1}{2} \frac{d}{d t}\left|w^{0}\right|_{L^{2}}^{2}, \\
-\nu \int_{\Omega} \Delta w^{0} \cdot w^{0} & =\nu\left|\nabla w^{0}\right|_{L^{2}}^{2} \\
\int_{\Omega}\left(u^{\nu} \cdot \nabla\right) w^{0} \cdot w^{0} & =0 \\
\left|\int_{\Omega}\left(w^{0} \cdot \nabla\right) u^{0} \cdot w^{0}\right| & \leq\left|\nabla u^{0}\right|_{L^{\infty}}\left|w^{0}\right|_{L^{2}}^{2} \leq \kappa\left|w^{0}\right|_{L^{2}}^{2} \\
\left|\int_{\Omega}\left(w^{0} \cdot \nabla\right) \theta^{0} \cdot w^{0}\right| & =\left|\int_{\Omega}\left(w^{0} \cdot \nabla\right) w^{0} \cdot \theta^{0}\right| \\
& \leq\left|\int_{\Omega}\left(w^{0} \cdot \nabla\right) w^{0} C_{00} \Phi\right|+\left|\int_{\Omega}\left(w^{0} \cdot \nabla\right) w^{0} \cdot \nu \widetilde{C}_{0}\right| \\
& \leq\left|\frac{w^{0}}{z}\right|_{L^{2}}\left|\nabla w^{0}\right|_{L^{2}}\left|C_{00} z \Phi\right|_{L^{\prime}}+\nu\left|w^{0}\right|_{L^{2}}\left|\nabla w^{0}\right|_{L^{2}}\left|\widetilde{C}_{0}\right|_{L^{\infty}} \\
& \leq \frac{\nu}{8}\left|\nabla w^{0}\right|_{L^{2}}^{2}+\kappa\left|w^{0}\right|_{L^{2}}^{2}+\frac{2 \nu}{e}\left|\frac{C_{00}}{\left.u_{3}^{0}\right|_{z=0}}\right|_{L^{\infty}} \cdot\left|\nabla w^{0}\right|_{L^{2}}^{2} .
\end{aligned}
$$

Thus under the relative smallness of the tangential slip at the boundary with respect to the suction i.e.

$$
\frac{2}{e}\left|\frac{C_{00}}{\left.u_{3}^{0}\right|_{z=0}}\right|_{L^{\infty}} \leq \frac{1}{8}
$$

we have

$$
\begin{aligned}
& \frac{1}{2} \frac{d}{d t}\left|w^{0}\right|_{L^{2}}^{2}+\frac{\nu}{2}\left|\nabla w^{0}\right|_{L^{2}}^{2} \leq \kappa\left|w^{0}\right|_{L^{2}}^{2}+\kappa \nu^{2}, \\
& w^{0}=u^{\nu}-u^{0}-\theta^{0}=0 \text { at } t=0,
\end{aligned}
$$

since we do not assume boundary layer at time zero, (i.e. $u^{\nu}=u^{0}=u_{0}$ at $t=0$ ). 
Hence under the assumption $*$ we deduce the following:

$$
\begin{aligned}
\left\|w^{0}\right\|_{L^{\infty}\left(0, T ; L^{2}(\Omega)\right)} & =\left\|u^{\nu}-u^{0}-\theta^{0}\right\|_{L^{\infty}\left(0, T ; L^{2}(\Omega)\right)} \leq \kappa \nu, \\
\left\|w^{0}\right\|_{L^{2}\left(0, T ; H_{0}^{1}(\Omega)\right)} & =\left\|u^{\nu}-u^{0}-\theta^{0}\right\|_{L^{2}\left(0, T ; H_{0}^{1}(\Omega)\right)} \leq \kappa \nu^{1 / 2} .
\end{aligned}
$$

This implies the validity of the Prandtl equation. For the physically more realistic uniform in space and time estimates, we could employ an anisotropic Sobolev imbedding and utilize a heuristic idea of better control on the tangential derivative of the velocity field than the normal derivative of the velocity field. For more details, the reader is referred to Temam and Wang (2000) for the two dimensional case. The three dimensional case is still unknown.

For the condition $(*)$, we notice that $C_{010}$ is directly related to $u_{\tau}^{0}(t, x, y, 0)-v_{\mathrm{bot}, \tau}(t, x, y)$. Also $u_{\tau}^{0}(0, x, y, 0)-v_{\text {bot }, \tau}(0, x, y)=,u_{0, \tau}(x, y, 0)-v_{\text {bot }, \tau}(0, x, y)=0$. Hence $(*)$ may be rewritten as

$$
\left|\frac{\left.u_{\tau}^{0}\right|_{z=0}-v_{\mathrm{bot}, \tau}}{\left.u_{3}^{0}\right|_{z=0}}\right|_{L^{\infty}} \leq \kappa_{0},
$$

and $(* *)$ is true for $t \leq T_{*}$ due to the well posedness of the inviscid problem and the fact that $u_{\tau}^{0}(0, x, y, 0)-v_{\text {bot }, \tau}(0, x, y)=0$. To summarize we have,

Theorem 3.1. There exists $T_{*}>0$ and $\kappa>0$ depending on the data but independent of the kinematic viscosity $\nu$ such that

$$
\begin{aligned}
& \left\|u^{\nu}-u^{0}-\theta^{0}\right\|_{L^{\infty}\left(0, T ; L^{2}(\Omega)\right)} \leq \kappa \nu, \\
& \left\|u^{\nu}-u^{0}-\theta^{0}\right\|_{L^{2}\left(0, T, H_{0}^{1}(\Omega)\right)} \leq \kappa \nu^{1 / 2}, \\
& \left\|u^{\nu}-u^{0}-\theta^{0}\right\|_{L^{\infty}((0, T) \times \Omega)} \leq \kappa \nu^{1 / 4} .
\end{aligned}
$$

Remark 3.1. The uniform in space and time estimate is not optimal. The optimal rate should be $\nu$ which is clear from our formal asymptotic expansion.

\section{Discussion ON STABILITY}

In this section we consider the stability issue for the boundary layer. The stability issue is intimately related to the question on whether the boundary layer derived in the previous sections is physically relevant (i.e. if it can actually appear).

As a special example of the laminar boundary layer we notice that

$$
v_{\infty}=\left(U_{1} \frac{e^{-U z / \nu}-e^{-U h / \nu}}{1-e^{-U h / \nu}}, 0,-U\right)
$$

is an exact steady solution to the Navier-Stokes equation with

$$
v_{\text {top }}=(0,0,-U)
$$


and

$$
v_{\text {bot }}=\left(U_{1}, 0,-U\right)
$$

and

$$
f \equiv 0,
$$

where $U_{1}$ and $U\left(=-U_{3}\right)$ are positive constants. The corresponding inviscid (steady) solution is

$$
(0,0,-U)
$$

Thus the relative smallness of horizontal shears with respect to the vertical suction can be reformulated as

$$
\frac{U_{1}}{U} \leq \frac{e}{16}
$$

The stability of $v_{\infty}$ under the smallness assumption can be derived using the usual energy method (see Ch. Doering, E. Spiegel, and R. Worthing (2000)). Indeed, let $v$ be the perturbation, $v=u-v_{\infty}$. Then $v$ satisfies the equation

$$
\begin{aligned}
\frac{\partial v}{\partial t}-\nu \Delta v+(u \cdot \nabla) v+(v \cdot \nabla) v_{\infty}+\nabla q & =0, \\
\operatorname{div} v & =0, \\
v=0 \text { at } z & =0 \text { and } h .
\end{aligned}
$$

Multiplying the equation by $v$ and integrating over the domain $\Omega$, utilizing the skew symmetry of the nonlinear term and the fact that

$$
\begin{aligned}
\left|\int_{\Omega}(v \cdot \nabla) v_{\infty} \cdot v\right| & =\left|-\int_{\Omega} v_{3} \frac{U_{1} U e^{-U z / \nu}}{\nu\left(1-e^{-U h / \nu}\right)} \cdot v_{1}\right| \\
& \leq\left|\frac{v_{1}}{z}\right|_{L^{2}}\left|\frac{v_{3}}{z}\right|_{L^{2}} \frac{U U_{1}}{\nu}\left|z^{2} e^{-U z / \nu}\right|_{L^{\infty}} \\
& \leq 2|\nabla v|_{L^{2}}^{2} \cdot \frac{U U_{1}}{\nu} \cdot \frac{\nu^{2}}{U^{2}} 4 e^{-2} \\
& =\frac{8 U_{1}}{e^{2} U} \nu|\nabla v|_{L^{2}}^{2} \\
& \leq \frac{\nu}{4}|\nabla v|_{L^{2}}^{2}
\end{aligned}
$$

provided the smallness condition holds, we have

$$
\frac{d}{d t}|v|_{L^{2}}^{2}+\nu|\nabla v|_{L^{2}}^{2} \leq 0
$$

which implies the asymptotic stability of the boundary layer. 
If the smallness condition is violated, in particular if the quantity $\frac{U_{1}}{U}$ is large, numerical evidence indicates that the boundary layer is linearly unstable (see Ch. Doering, E. Spiegel, and R. Worthing (2000)).

Another indicator of the stability of the boundary layer derived in the previous sections is the continuous dependence of the solutions on the initial data and external forcing independent of the Reynolds number, under the smallness assumption (or short-time assumption). This is not trivial even if we have the well-posedness of the Navier-Stokes system. The usual well-posedness indicates that for each $\varepsilon>0$, there exists $\delta>0$, such that if the difference in initial data is less than $\delta$, then the solutions will differ by no more than $\varepsilon$ within the time period $[0, T]$. Usually $\delta$ depends on all data including the kinematic viscosity and the solution that we chose. Here we show that $\delta$ can be chosen independent of the kinematic viscosity $\nu$ provided that the time is small enough so that the relative smallness of tangential shear with respect to the vertical suction holds.

More precisely we have, for $u^{\nu}$, an exact solution satisfying the estimates in $\S 3$, i.e.

$$
\begin{aligned}
& \left\|u^{\nu}-u^{0}-\theta^{0}\right\|_{L^{\infty}\left(0, T ; L^{2}\right)} \leq \kappa \nu \\
& \left\|u^{\nu}-u^{0}-\theta^{0}\right\|_{L^{2}\left(0, T ; H_{0}^{1}\right)} \leq \kappa \nu^{1 / 2} \\
\left\|z \theta^{0}\right\|_{L^{\infty}} \leq & \frac{\nu}{8}
\end{aligned}
$$

and for any $\varepsilon>0$, there exists $\delta>0$, independent of $\nu$ such that

$$
\left\|v^{\nu}-u^{\nu}\right\|_{L^{\infty}\left(0, T ; L^{2}\right)} \leq \varepsilon
$$

provided

$$
\left\|v_{0}-u_{0}\right\|_{L^{2}} \leq \delta
$$

where $v^{\nu}$ is the solution to the Navier-Stokes system with initial data $v_{0}$.

We invoke energy method to prove this uniform continuous dependence result.

Let $w^{\nu}=v^{\nu}-u^{\nu}$; then we have

$$
\begin{aligned}
\frac{\partial w^{\nu}}{\partial t}-\nu \Delta w^{\nu}+\left(v^{\nu} \cdot \nabla\right) w^{\nu}+\left(w^{\nu} \cdot \nabla\right) u^{\nu}+\nabla q & =0, \\
\operatorname{div} w^{\nu} & =0, \\
w^{\nu} & =0 \text { at } z=0 \text { and } h .
\end{aligned}
$$


Multiplying the $w^{\nu}$ equation by $w^{\nu}$, integrating over $\Omega$ and utilizing the skew symmetry of the nonlinear term we find

$$
\begin{aligned}
\frac{1}{2} \frac{d}{d t}\left|w^{\nu}\right|_{L^{2}}^{2}+\nu\left|\nabla w^{\nu}\right|_{L^{2}}^{2} \leq & -\int_{\Omega}\left(w^{\nu} \cdot \nabla\right) u^{\nu} \cdot w^{\nu} \\
= & -\int_{\Omega}\left(w^{\nu} \cdot \nabla\right) u^{0} \cdot w^{\nu} \\
& +\int_{\Omega}\left(w^{\nu} \cdot \nabla\right) w^{\nu} \cdot \theta^{0} \\
& +\int_{\Omega}\left(w^{\nu} \cdot \nabla\right)\left(u^{\nu}-u^{0}-\theta^{0}\right) \cdot w^{\nu} \\
\leq & \left|\nabla u^{0}\right|_{L^{\infty}}\left|w^{\nu}\right|_{L^{2}}^{2} \\
& +\left.\left|\frac{w^{\nu}}{z}\right|_{L^{2}}\left|\nabla w_{L^{2}}^{\nu}\right| z \theta^{0}\right|_{L^{\infty}} \\
& +\left|\nabla\left(u^{\nu}-u^{0}-\theta^{0}\right)\right|_{L^{2}}\left|w^{\nu}\right|_{L^{4}}^{2} \\
\leq & \kappa\left|w^{\nu}\right|_{L^{2}}^{2}+\frac{\nu}{4}\left|\nabla w^{\nu}\right|_{L^{2}}^{2}+\kappa \nu^{1 / 2}\left|w^{\nu}\right|_{L^{2}}\left|\nabla w^{\nu}\right|_{L^{2}} \\
\leq & \kappa\left|w^{\nu}\right|_{L^{2}}^{2}+\frac{\nu}{2}\left|\nabla w^{\nu}\right|_{L^{2}}^{2} .
\end{aligned}
$$

Hence we have

$$
\begin{aligned}
\frac{d}{d t}\left|w^{\nu}\right|_{L^{2}}^{2}+\nu\left|\nabla w^{\nu}\right|_{L^{2}}^{2} & \leq \kappa\left|w^{2}\right|_{L^{2}}^{2} \\
\left\|w^{\nu}\right\|_{L^{\infty}\left(0, T ; L^{2}(\Omega)\right)} & \leq e^{\frac{1}{2} \kappa T}\left\|w_{0}\right\|_{L^{2}} \\
& =e^{\frac{1}{2} \kappa T}\left\|u_{0}-v_{0}\right\|_{L^{2}}
\end{aligned}
$$

The stability then holds by taking

$$
\delta=e^{-\frac{1}{2} \kappa T} \varepsilon
$$

\section{APPENDIX}

We consider solutions to the Prandtl type equation at order -1. For the sake of exposition we consider the special case of a two dimensional flow (with $y$ suppressed) with $\left.u_{3}^{0}\right|_{z=0}=-U$ ( $U>0$ a fixed constant). The Prandtl type equation at order -1 is

$$
\begin{aligned}
-\nu \frac{\partial^{2} \theta^{0}}{\partial z^{2}}-U \frac{\partial \theta^{0}}{\partial z}+\nabla q^{0} & =0 \text { in } \Omega \\
\operatorname{div} \theta^{0} & =0 \text { in } \Omega \\
\theta^{0} & =0 \text { at } z=h \\
\theta & =\left(-\left.u_{1}^{0}\right|_{z=0}, 0\right) \text { at } z=0 .
\end{aligned}
$$


Suppose that $-\left.u_{1}^{0}\right|_{z=0}$ has the following Fourier expansion

$$
-\left.u_{1}^{0}\right|_{z=0}=\sum_{k} b_{k}(t) e^{2 k \pi i x / L_{1}}
$$

We invoke the stream function formulation for the corrector $\theta^{0}$,

$$
\begin{aligned}
\theta^{0}= & \sum_{k \neq 0} \operatorname{curl}\left(\varphi_{k}(t, z) e^{2 k \pi i x / L_{1}}\right) \\
& +b_{0}(t)\left(\frac{e^{-U z / \nu}-e^{-U h / \nu}}{1-e^{-U h / \nu}}, 0\right) \\
= & \sum_{k \neq 0}\left(\frac{\partial \varphi k}{\partial z}(t, z),-2 \pi k i \varphi_{k}(t, z) e^{2 \pi k i x / L_{1}}\right) \\
& +b_{0}(t)\left(\frac{e^{-U z / \nu}-e^{-U h / \nu}}{1-e^{-U h / \nu}}, 0\right)
\end{aligned}
$$

For $k \neq 0$, the $\varphi_{k}$ 's satisfy the equations

$$
\begin{aligned}
-\nu\left(\frac{\partial^{4} \varphi_{k}}{\partial z^{4}}-\frac{4 \pi^{2} k^{2}}{L_{1}^{2}} \frac{\partial^{2} \varphi_{k}}{\partial z^{2}}\right)-U\left(\frac{\partial^{3} \varphi_{k}}{\partial z^{3}}-\frac{4 \pi^{2} k^{2}}{L_{1}^{2}} \frac{\partial \varphi_{k}}{\partial z}\right) & =0, \\
\varphi_{k}(t, h)=\varphi_{k}(t, 0) & =0, \\
\frac{\partial \varphi_{k}}{\partial z}(t, h) & =0, \\
\frac{\partial \varphi_{k}}{\partial z}(t, 0) & =b_{k}(t) .
\end{aligned}
$$

The equation can be written as

$$
\frac{\partial \varphi_{k}}{\partial z}\left(\frac{\partial \varphi_{k}}{\partial z}+\frac{U}{\nu}\right)\left(\frac{\partial \varphi_{k}}{\partial z}-\frac{2 \pi k}{L_{1}}\right)\left(\frac{\partial \varphi_{k}}{\partial z}+\frac{2 \pi k}{L_{1}}\right)=0 .
$$

In the case without resonance, i.e. $\frac{U}{\nu} \neq \pm \frac{2 \pi k}{L_{1}}$ the solutions must take the form

$$
\varphi_{k}(t, z)=b_{k}(t)\left(C_{k 0}+C_{k 1} e^{2 \pi k z / L_{1}}+C_{k 2} e^{-2 \pi k z / L_{1}}+C_{k_{3}} e^{-U z / \nu}\right)
$$

Notice that the coefficient $C_{k 0}, C_{k 1}, C_{k 2}, C_{k 3}$ are independent of $b_{k}(t)$. Assuming sufficient regularity on $u^{0}$ which translates into the smallness of $b_{k}(t)$ for large $k$ and the exact formula for the solution satisfying the boundary condition we see that

$$
\sum_{k \neq \pm \frac{U}{\nu} \frac{L_{1}}{2 \pi}} \operatorname{curl}\left(\varphi_{k}(t, z) e^{2 \pi k i x / L_{1}}\right)
$$


takes the form

$$
C_{0}(t ; x) e^{-U / \nu z}+\nu \widetilde{C}_{0}(t, x, z) .
$$

In the case of resonance, say $\frac{U}{\nu}=\frac{2 \pi k}{L_{1}}$ the solution for the Fourier coefficients of the stream function takes the form

$$
\varphi_{k}(t, z)=b_{k}(t)\left(C_{k 0}+C_{k 1} e^{2 k \pi z / L_{1}}+\left(C_{k 2}+C_{k 3} z\right) e^{-2 k \pi z / L_{1}}\right)
$$

since $k=\frac{U}{\nu} \cdot \frac{L_{1}}{2 \pi}, b_{k}$ is extremely small. Thus curl $\left(\varphi_{k}(t, z) e^{2 \pi k x / L_{1}}\right)$ still satisfy the same kind of estimates.

\section{ACKNOWLEDGMENTS}

The work of Temam was supported in part by the National Science Foundation grant NSF-DMS-9705229 and by the Research Fund of Indiana University. The work of Wang was supported in part by the National Science Foundation grant NSF-DMS-9971986, a Start Up Fund, a Faculty Development Fund from the Iowa State University and the Institute of Mathematical Sciences at the Chinese University of Hong Kong.

\section{REFERENCES}

[1] S.N. Alekseenko, Existence and Asymptotic Representation of Weak Solutions to the Flowing Problem Under the Condition of Regular Slippage on Solid Walls, Siberian Mathematical Journal, Vol.35, No.2, (19 94), 209-230.

[2] S.N. Antontsev, A.V. Kazhikhov and V.N. Monakhov, Boundary Value Problems in Mechanics of Nonhomogeneous Fluids, North-Holland (1990).

[3] R. Balian and J. L. Peube, Fluid dynamics, Cours de l'Ecole d'Eté de Physique Théorique, Les Houches, Gordon and Breach Science Publishers, New-York (1977).

[4] G. K. Batchelor, An Introduction to Fluid Dynamics, Cambridge University Press, Cambridge (1967).

[5] P. Constantin and J. Wu, Inviscid limit for vortex patches, Nonlinearity 8 (1995)), 735-742.

[6] Ch.R. Doering, E.A. Spiegel And R.A. Worthing, Laminar and turbulent energy dissipation in a shear boundary layer with suction, to appear in Physics of Fluids.

[7] T. KATO, Remarks on the zero viscosity limit for nonstationary Navier-Stokes flows with boundary, in Seminar on PDE, edited by S.S.Chern, Springer, N.Y., (1984).

[8] O.A. Ladyzhenskaya, book, 1962 see in 3. The mathematical theory of viscous incompressible flows, 2nd ed. Gordon and Breach, New York, (1969).

[9] P. Lagerström, Matched Asymptotics Expansion, Ideas and Techniques, Springer-Verlag, New York, (1988).

[10] H. LAmB, Hydordynamics, reprinted by Dover, New-York, (1945).

[11] J.L. Lions, Perturbations singulières dans les problèmes aux limites et en controle optimal, Lecture Notes in Math., vol 323, Springer-Verlag, New York, (1973).

[12] J.L. Lions, Méthodes de Résolution des Problèmes aux Limites Non Linéaires, Dunod, Paris, (1969).

[13] H. K. Moffatt, Six lectures on general fluid dynamics and two on hydromagnetic dynamo theory. In $[1]$.

[14] O. A. Oleinik, The Prandtl system of equations in boundary layer theory, Dokl. Akad. Nauk S.S.S.R. $150,4,(3),(1963), 583-586$. 
[15] O. A. Oleinik And V. N. Samokhin, Mathematical models in boundary layer theory, Applied Mathematics and Mathematical Computation, 15, Chapman \& Hall/CRC, Boca Raton, FL 1999.

[16] L. PRAndtL, Verber Flüssigkeiten bei sehr kleiner Reibung, Verk. III Intem. Math. Kongr. Heidelberg (1905), 484-491, Teuber, Leibzig.

[17] L. RosenheAD, Laminar boundary layers; an account of the development, structure, and stability of laminar boundary layers in incompressible fluids, together with a description of associated experimental techniques, Oxford Clarendon Press, (1963).

[18] M. SAmmartino AND R. E. CAFLiSCH, Zero viscosity limit for analytic solutions of the Navier-Stokes equations. Proceedings of the VIII International Conference on Waves and Stability in Continuous Media, Part II (Palermo, 1995). Rend. Circ. Mat. Palermo

[19] R. Temam, Navier-Stokes Equations, North-Holland, 3rd Edition, Amsterdam, (1984).

[20] R. Temam, Behaviour at time $t=0$ of the solutions of semi-linear evolution equations, J. Diff. Equ., 17, (1982), 73-92.

[21] R. Temam, Navier-Stokes Equations and Nonlinear Functional Analysis, CBMS-NSF Regional Conference Series in Applied MAth., SIAM, Philadelphia, (1983).

[22] R. Temam And X. WAng, Asymptotic analysis of the linearized Navier-Stokes equations in a channel, Differential and Integral Equations, 8, no. 7, (1995), 1591-1618.

[23] R. Temam and X. Wang, Asymptotic Analysis of Oseen Type Equations in a Channel at Small Viscosity, Indiana University Mathematics Journal, 45, (1996), 863-916.

[24] R. TEMAM AND X. WANG, On the behavior of the solutions of the Navier-Stokes equations at vanishing viscosity, Annali della Scuola Normale Superiore de Pisa, volume dedicated to the memory of E. De Giorgi, Serie IV, Vol. XXV (1998), 807-828.

[25] R. Temam And X. Wang, Asymptotic analysis of the linearized Navier-Stokes equations in a general $2 D$ domain, Asymptotic Analysis, 9, (1997), 1-30.

[26] R. Temam And X. WAng, Boundary Layers Associated with Incompressible Navier-Stokes Equations: the Noncharacteristic Boundary Case (2000), to appear.

[27] M. van Dyke, An album of fluid motion, Parabolic Press, Stanford, California, (1982).

[28] M. I. VishiK AND L. A. LyUSTERnik, Regular degeneration and boundary layer for linear differential equations with small parameter, Uspekki Mat. Nauk, vol 12, (1957), 3-122.

[29] R. von Mises And K.O. Friedrichs, Fluid Dynamics, Springer-Verlag New York, Heidelberg, Berlin, (1971). 